\title{
Efficacy and safety of ethanol infusion into the vein of Marshall for mitral isthmus ablation
}

\author{
Anna Lam ${ }^{1}$, Thomas Küffer ${ }^{1}$, Lukas Hunziker ${ }^{1}$, Nikolas Nozica ${ }^{1}$, Babken Asatryan ${ }^{1}$, \\ Florian Franzeck ${ }^{1}$, Antonio Madaffari ${ }^{1}$, Andreas Haeberlin ${ }^{1}$, Aline Mühl ${ }^{1}$, Helge Servatius ${ }^{1}$, \\ Jens Seiler ${ }^{1}$, Fabian Noti ${ }^{1}$, Samuel Baldinger ${ }^{1}$, Hildegard Tanner ${ }^{1}$, Stephan Windecker ${ }^{1}$, \\ Tobias Reichlin ${ }^{1}$, and Laurent Roten ${ }^{1}$ \\ ${ }^{1}$ Inselspital University Hospital Bern
}

February 20, 2021

\begin{abstract}
Introduction: Chemical ablation by retrograde infusion of ethanol into the vein of Marshall (VOM-EI) can facilitate achievement of mitral isthmus block. This study sought to describe efficacy and safety of this technique. Methods and Results: Twenty-two consecutive patients (14 male, median age 71 years) with attempted VOM-EI for mitral isthmus ablation were included in the study. VOM-EI was successfully performed with a median of $4 \mathrm{ml}$ of $96 \%$ ethanol in 19 patients (86\%) and mitral isthmus was successfully blocked in all (100\%). Touch up endocardial and/or epicardial ablation after VOM-EI was necessary in 12 patients $(63 \%)$. Perimitral flutter was present in 12 patients $(63 \%)$ during VOM-EI and terminated or slowed by VOM-EI in four and three patients, respectively. Low-voltage area of the mitral isthmus region increased from $3.1 \mathrm{~cm} 2$ (IQR 0-7.9) before to $13.2 \mathrm{~cm} 2$ (IQR 8.2-15.0) after VOM-EI and correlated significantly with the volume of ethanol injected $(\mathrm{P}=0.03)$. Median high-sensitive cardiac troponin- $\mathrm{T}$ increased significantly from $330 \mathrm{ng} / \mathrm{L}$ (IQR 221-516) the evening of the procedure to $598 \mathrm{ng} / \mathrm{L}$ (IQR 382-769; $\mathrm{P}=0.02$ ) the following morning. A small pericardial effusion occurred in three patients (16\%), mild pericarditis in one (5\%) and uneventful VOM dissection in two (11\%). After a median follow-up of 3.5 months (IQR 3.0-11.0), 10 of 18 patients $(56 \%)$ with VOM-EI and available follow-up had arrhythmia recurrence. Repeat ablation was performed in five patients $(50 \%)$ and peri-mitral flutter diagnosed in three $(60 \%)$. Conclusion: VOM-EI is feasible, safe and effective to achieve acute mitral isthmus block
\end{abstract}

\section{Efficacy and safety of ethanol infusion into the vein of Marshall for mitral isthmus ablation}

Anna Lam MD | Thomas Küffer MSc | Lukas Hunziker MD | Nikolas Nozica MD | Babken Asatryan MD, PhD | Florian Franzeck MD | Antonio Madaffari, MD | Andreas Haeberlin MD, PhD | Aline Mühl MSc | Helge Servatius MD | Jens Seiler MD | Fabian Noti MD | Samuel H. Baldinger MD | Hildegard Tanner MD | Stephan Windecker MD | Tobias Reichlin MD | Laurent Roten MD

\section{All authors}

Department of Cardiology, Inselspital, Bern University Hospital and University of Bern, Bern, Switzerland

\section{Corresponding author}

Laurent Roten, MD, MBA, Inselspital, Kardiologie, Freiburgstrasse, 3010 Bern, Switzerland

Email: laurent.roten@insel.ch

Phone: 41 (0) 316325263

\section{CONFLICT OF INTEREST}


Haeberlin A. is Co-founder and CEO of Act-Inno AG, consultant of Cairdac and has received travel/educational grants from Medtronic and Spectranetics/Philips.

The spouse of Seiler J. is an employee of Boston Scientific.

Noti F. has received travel grant from Abbott, Medtronic, Boston Scientific and Spectranetics.

Tanner H. has received travel grant from Abbott and educational grant from Biosense Webster.

Windecker St. reports research and educational grants to the institution from Abbott, Amgen, BMS, Bayer, Boston Scientific, Biotronik, Cardinal Health, CardioValve, CSL Behring, Daiichi Sankyo, Edwards Lifesciences, Johnson\&Johnson, Medtronic, Querbet, Polares, Sanofi, Terumo, Sinomed.

Windecker St. serves as unpaid advisory board member and/or unpaid member of the steering/executive group of trials funded by Abbott, Abiomed, Amgen, Astra Zeneca, BMS, Boston Scientific, Biotronik, Cardiovalve, Edwards Lifesciences, MedAlliance, Medtronic, Novartis, Polares, Sinomed, V-Wave and Xeltis, but has not received personal payments by pharmaceutical companies or device manufacturers. He is also member of the steering/excecutive committee group of several investigated-initiated trials that receive funding by industry without impact on his personal remuneration. Windecker St. is an unpaid member of the Pfizer Research Award selection committee in Switzerland.

Reichlin T. has received speaker/consulting honoraria or travel support from Abbott/SJM, Astra Zeneca, Brahms, Bayer, Biosense-Webster, Medtronic, Pfizer-BMS and Roche and he has received support for his institution's fellowship program from Biosense-Webster, Biotronik, Medtronic, Abbott/SJM and Boston Scientific.

Roten L. has received speaker honoraria from Abbott and Medtronic. The other authors have no potential conflict of interest relevant to this article.

\section{FUNDING}

None

\section{Abstract}

Introduction: Chemical ablation by retrograde infusion of ethanol into the vein of Marshall (VOM-EI) can facilitate achievement of mitral isthmus block. This study sought to describe efficacy and safety of this technique.

Methods and Results: Twenty-two consecutive patients (14 male, median age 71 years) with attempted VOM-EI for mitral isthmus ablation were included in the study. VOM-EI was successfully performed with a median of $4 \mathrm{ml}$ of $96 \%$ ethanol in 19 patients (86\%) and mitral isthmus was successfully blocked in all (100\%). Touch up endocardial and/or epicardial ablation after VOM-EI was necessary in 12 patients (63\%). Perimitral flutter was present in 12 patients $(63 \%)$ during VOM-EI and terminated or slowed by VOM-EI in four and three patients, respectively. Low-voltage area of the mitral isthmus region increased from $3.1 \mathrm{~cm}^{2}$ (IQR 0-7.9) before to $13.2 \mathrm{~cm}^{2}$ (IQR 8.2-15.0) after VOM-EI and correlated significantly with the volume of ethanol injected $(\mathrm{P}=0.03)$. Median high-sensitive cardiac troponin- $\mathrm{T}$ increased significantly from $330 \mathrm{ng} / \mathrm{L}$ (IQR 221-516) the evening of the procedure to $598 \mathrm{ng} / \mathrm{L}$ (IQR 382-769; $\mathrm{P}=0.02$ ) the following morning. A small pericardial effusion occurred in three patients (16\%), mild pericarditis in one (5\%) and uneventful VOM dissection in two (11\%). After a median follow-up of 3.5 months (IQR 3.0-11.0), 10 of 18 patients $(56 \%)$ with VOM-EI and available follow-up had arrhythmia recurrence. Repeat ablation was performed in five patients (50\%) and peri-mitral flutter diagnosed in three $(60 \%)$.

Conclusion: VOM-EI is feasible, safe and effective to achieve acute mitral isthmus block.

\section{KEYWORDS}

vein of Marshall, ligament of Marshall, atrial fibrillation, atrial tachycardia, ethanol infusion 


\section{1 | INTRODUCTION}

Endocardial radiofrequency catheter ablation of the mitral isthmus is commonly performed for peri-mitral reentrant tachycardia or as part of ablation strategy for persistent atrial fibrillation. ${ }^{1,2}$ Ablation of the mitral isthmus is challenging due to the anatomical complexity and the thickness of the tissue in this region, and because of epicardial cooling by the coronary sinus (CS) and circumflex artery. ${ }^{3}$ With both endocardial and epicardial ablation from inside the CS, acute complete bi-directional block of the mitral isthmus is achieved in only about $70 \%$ of patients. ${ }^{4,5}$

The ligament of Marshall is an epicardial vestigial fold that contains the vein of Marshall (VOM) and the Marshall bundle. ${ }^{6}$ The VOM drains into the CS and runs posteriorly and superiorly along the epicardial surface of the left atrium, to join the anterior aspect of the left-sided pulmonary veins. Myofibers of the VOM can form an epicardial bridge over the mitral isthmus, preventing successful ablation from the endocardium and from within the CS. ${ }^{7}$

In 2009, Valderrabano et al. first described chemical ablation of the VOM by retrograde infusion of ethanol into the VOM (VOM-EI) and showed that VOM-EI can facilitate mitral isthmus ablation. ${ }^{8}{ }^{9}$ Subsequently, other groups adopted and refined the technique of VOM-EI for mitral isthmus ablation with high success rates. ${ }^{10}$ Linear ablation of the mitral isthmus is an additional target of ablation of persistent atrial fibrillation, and VOM-EI may be used in these patients. The recent VENUS-trial, a prospective multicentre randomized study, showed improved arrhythmia-free outcome in patients with persistent atrial fibrillation treated with catheter ablation with supplement ethanol infusion into the vein of Marshall. ${ }^{11}$

The aim of the present study was to describe our single-centre experience of efficacy and safety of VOM-EI for mitral isthmus ablation.

\section{2 | METHODS}

Since June 2019, we use ancillary VOM-EI for mitral isthmus ablation. We perform mitral isthmus ablation for peri-mitral flutter or as part of an ablation strategy to treat persistent atrial fibrillation resistant to pulmonary vein isolation, if peri-mitral flutter is suspected to be a perpetuating factor. Consecutive patients undergoing VOM-EI until August 2020 were included in this study. All patients undergoing atrial fibrillation ablation at our centre are included in a prospective registry. This registry has been approved by the local ethics committee. All patients provided written informed consent to participate in the registry.

\section{1 | Ablation procedure}

Prior to the ablation procedure, cardiac computed tomography and transesophageal echocardiography were performed in all patients to define left atrial anatomy, and to rule out intracardiac thrombus. After obtaining venous access, a steerable, decapolar catheter (Dynamic XT, Boston Scientific, Marlborough, MA) was positioned within the CS. Left atrial access was gained by transseptal puncture or via a patent foramen ovale. Heparin was administered to achieve an activated clotting time of more than 350 seconds. An electroanatomical 3D mapping system (CARTO 3, Biosense Webster, Diamond Bar, CA) was used in all cases and left atrial geometry reconstructed with a multipolar mapping catheter (Pentaray, Biosense Webster, Diamond Bar, CA). Left atrial voltage maps were generated before and after VOM-EI and low-voltage area was defined as bipolar voltage amplitude below $0.5 \mathrm{mV}$ (Figure 1). Radiofrequency ablation of the mitral isthmus was performed endocardially and from within the CS as necessary to achieve bi-directional block.

For ablation, an irrigated-tip ablation catheter (ThermoCool SmartTouch SF, Biosense Webster, Diamond Bar, CA) was used with a power of $20-25 \mathrm{~W}$ in the $\mathrm{CS}$ and $35 \mathrm{~W}$ in the atrium. Bi-directional block was confirmed by differential pacing on both sides of the mitral isthmus according to standard criteria. ${ }^{1}$ Radiofrequency ablation of targets other than the mitral isthmus was performed at the discretion of the operator and as mandated by the clinical situation.

\section{2 | Vein of Marshall ethanol infusion (VOM-EI)}


The technique of VOM-EI has been described elsewhere. ${ }^{10}$ In brief, we cannulated the CS with an 8.5 Fr steerable sheath (Agilis NXT, Abbott Inc, St. Paul, MN) and placed a 6-Fr left internal mammary artery (LIMA) guiding catheter into the great cardiac vein. In a right anterior oblique view, we performed angiography to localize the ostium of the VOM. If a suitable VOM was present, we positioned on an overthe-wire percutaneous coronary angioplasty balloon (Emerge 1.5-2.5 mm x $8 \mathrm{~mm}$; Boston Scientific) into the proximal VOM advanced over a preloaded coronary guide-wire (Sion Blue, Asahi Intecc USA, Inc, Santa Ana, CA). After balloon inflation, we removed the guidewire and obtained a selective VOM angiography by injecting contrast through the wire port of the balloon. After ensuring proximal balloon occlusion and adequate VOM angiography, we slowly injected 96\% ethanol into the VOM over 1-2 minutes. During VOMEI, we monitored the cycle length of peri-mitral flutter in patients with ongoing tachycardia. In case of sinus rhythm, we paced the left atrium from the left atrial appendage and measured the delay on the decapolar catheter placed over the mitral isthmus via the great cardiac vein. After VOM-EI, we performed selective VOM angiography to document chemical ablation of the VOM.

The following day, we recorded a 12-lead-ECG in all patients and performed transthoracic echocardiography to rule out pericardial effusion. We obtained cardiac biomarkers the evening of the procedure and the following morning.

\section{3 | Follow-up and redo procedure}

Patients were followed up after 3, 6 and 12 months. During follow-up, a 7-day Holter ECG was recorded. Arrhythmia recurrence was defined as atrial fibrillation, atrial tachycardia or atrial flutter on a 12-lead ECG or on a rhythm strip with [?] 30 seconds duration, after a blanking period of 3 months. Patients were offered a redo procedure as deemed clinically appropriate. At the beginning of all redo procedures, an electroanatomical 3D voltage map of the left atrium was generated and mitral isthmus block reassessed.

\section{4 | Statistical analysis}

Continuous variables are presented as median with interquartile range (IQR $25^{\text {th }}-75^{\text {th }}$ percentile). Categorical variables are expressed as numbers and percentages. Continuous variables were compared using the ANOVA test or the Mann-Whitney U test, as appropriate. Spearman's correlation test was used to test for any associations between two categorical variables. All reported $\mathrm{P}$ values are two-sided, with a $\mathrm{P}<$ 0.05 considered to indicate statistical significance. Statistical analyses were performed using the Statistical Package for Social Studies (SPSS), version 25.0 (IBM, Armonk, NY).

\section{3 | RESULTS}

\section{1 | Patient characteristics}

We attempted VOM-EI in 22 patients (14 male [64\%]) with a median age 71 years (IQR 55-74). Patient characteristics and details of previous ablation procedures are summarised in Table 1. In four patients $(22 \%)$, radiofrequency ablation of the mitral isthmus had been attempted in a previous procedure and was not successful. Four patients had no previous left atrial ablation procedures.

\subsection{Procedural characteristics}

Vein of Marshall ethanol infusion was successfully performed in 19 patients (86\%). VOM-EI was not performed in three patients due to a missing VOM, a too small VOM diameter for cannulation, and a VOM dissection during cannulation. Among patients with successful VOM-EI, radiofrequency ablation of the mitral isthmus was performed during the same procedure before VOM-EI in 8 patients (42\%), without achieving mitral isthmus block. Ancillary radiofrequency ablation during the VOM-EI procedure included pulmonary vein isolation in 11 patients (58\%), roof line ablation in 8 (42\%), left atrial defragmentation in one (5\%), superior vena cava isolation in two (11\%), and cavotricuspid isthmus ablation in 4 patients $(21 \%)$.

During VOM-EI, a median of $4.0 \mathrm{ml}$ (IQR 3.0-6.0) 96\% ethanol was infused into the VOM. Perimitral flutter was present during VOM-EI in 12 patients $(63 \%)$ with termination during chemical ablation in $4(33 \%$; Figure 4$)$, and cycle length prolongation in $3(25 \%)$. Chemical ablation directly induced bidirectional mitral 
isthmus block in 7 of 19 patients (37\%). In the remaining 12 patients (63\%) bidirectional mitral isthmus block was successfully achieved with ancillary radiofrequency catheter ablation, for which we targeted the endocardial, annular side of the mitral isthmus in 11 patients $(92 \%)$ and the pulmonary venous side in 3 patients $(25 \%)$. Ancillary radiofrequency catheter ablation within the CS was performed in six patients (50\%) after VOM-EI.

The median low-voltage area in the lateral left atrium increased significantly from $3.1 \mathrm{~cm}^{2}$ (IQR 0-7.9) before to $13.2 \mathrm{~cm}^{2}$ (IQR 8.2-15.0; $\left.\mathrm{P}=0.02\right)$ after VOM-EI. Low-voltage area induced by VOM-EI correlated significantly with the volume of ethanol injected $(\mathrm{P}=0.03$; Figure 5$)$.

Creatine kinase (CK) and creatine kinase myocardial band (CK-MB) were 124 U/L (IQR 101-302) and 7.3 $\mu \mathrm{g} / \mathrm{L}$ (IQR 5.7-10.3), respectively, on the evening of the procedure, without significant change in the next morning (141 U/L [IQR 89-293] and $8.2 \mu \mathrm{g} / \mathrm{L}$ [IQR 4.5-9.5], respectively; Figure 6). High-sensitive cardiac troponin-T (hs-TnT) measured $330 \mathrm{ng} / \mathrm{L}$ (IQR 221-516) the evening of the procedure with a significant increase the following morning (598 ng/L [IQR 382-769]; $\mathrm{P}=0.02)$.

\section{3 | Adverse events}

We observed a small, clinically non-relevant pericardial effusion in three patients (16\%) on transthoracic echocardiography the day after the procedure, all of which resolved without additional interventions. One additional patient (5\%) complained about symptoms compatible with mild pericarditis. In one patient (5\%), a dual chamber pacemaker was implanted the day following the procedure because of symptomatic sinus arrest. Two VOMs were present in one patient (Figure 7). Cannulation of the smaller VOM resulted in uneventful dissection and VOM-EI of the larger VOM was successful. In another patient, a VOM with a normal diameter dissected during cannulation. Repeat cannulation of the VOM and VOM-EI was successful without any complications at the end of the ablation procedure.

\section{4 | Follow-up and redo procedures}

Follow-up was available in 18 patients (95\%) a median of 3.5 months (IQR 3.0-11.0) after VOM-EI. One patient was lost of follow-up. Ten patients (56\%) had recurrence of arrhythmia. Arrhythmia recurred as atrial fibrillation in six patients $(60 \%)$ and as atrial flutter in four $(40 \%)$. A repeat ablation procedure was performed in five patients (50\%). Among these, a peri-mitral flutter was diagnosed in three $(60 \%)$. The gaps in the mitral isthmus line located to the annular side in two patients and on both the annular and pulmonary venous side in the third patient. Re-ablation was performed endocardially in all three patients and within the CS in two patients, with induction of complete mitral isthmus block in all patients.

In two patients (40\%), the mitral isthmus was still blocked during the redo procedure.

The median low-voltage area in the lateral left atrium during the redo procedure was smaller compared to the low-voltage area at the end of the index procedure $\left(13.2 \mathrm{~cm}^{2}\right.$ [IQR 8.2-15.0] versus $10.5 \mathrm{~cm}^{2}$ [IQR 6.5-14.9], respectively; $\mathrm{P}=0.345$; Figure 8).

\section{4 | DISCUSSION}

Our main findings are:

1. VOM-EI was feasible in 19 of 22 patients $(86 \%)$ without major adverse events.

2. VOM-EI with ancillary radiofrequency ablation as necessary achieved mitral isthmus block in all patients.

3. The volume of ethanol injected into the VOM correlated with the area of scarring in the voltage map.

\subsection{Feasibility and safety of VOM-EI}

Hwang et al. first described the cannulation of the VOM in humans with a microelectrode catheter inserted via the femoral vein for signal analysis. ${ }^{12}$ Later, Valderrabano et al. used a right jugular vein approach for the first procedures of VOM-EI reported in humans and described a success rate of $86 \%{ }^{7}$ Kitamura et al. adapted the technique for femoral access using a LIMA guiding catheter introduced into a steerable sheath, 
with successful injection of ethanol in $92.6 \%$ of cases. ${ }^{10}$ Other groups reported successful VOM-EI rates of $73.4-92 \% .{ }^{11,13,14}$. Using the same approach, we successfully achieved VOM-EI in comparable $86 \%$ of cases.

Reported complications of VOM-EI include vascular access complications, pericardial effusion, pericarditis, VOM dissection and volume overload. ${ }^{10,11,13}$ In our population of patients with intended VOM-EI, we observed pericardial effusion/pericarditis in four patients (18\%) and VOM dissection in two (9\%), all of which resolved without additional interventions. We performed echocardiography the day after VOM-EI in $86 \%$ of patients, irrespective of symptom status. This strategy may have increased the incidence of clinically irrelevant pericardial effusion compared to other groups not performing routine echocardiography after VOM-EI.

The recent VENUS trial randomized patients to VOM-EI versus conventional radiofrequency ablation for mitral isthmus ablation. ${ }^{11}$ Overall complication rate was not different between groups. Of 185 patients randomized to VOM-EI, two patients suffered intraprocedural pericardial effusion and two additional patients had a subacute pericardial effusion requiring drainage. Another 11 patients had subacute pericardial effusion/pericarditis not necessitating drainage. Vascular access complications were present in three patients, stroke/TIA in three and volume overload in ten. Nakashima et al. performed VOM-EI in 152 patients and reported CS or VOM dissection in two cases and no other major adverse events. ${ }^{13}$ Another study including 84 patients for VOM-EI reported pericardial effusion without intervention in two patients and groin hematoma in three. ${ }^{14}$ No complications were described among 32 patients undergoing VOM-EI in the study by Liu et al. ${ }^{15}$

\subsection{Efficacy of VOM-EI}

In our study, we achieved mitral isthmus block in all patients undergoing VOM-EI. Other groups described comparable results of mitral isthmus block in $98.7-100 \%$ of cases. ${ }^{10,13,16}$

Importantly, ancillary radiofrequency ablation was necessary in $63 \%$ of our patients, mainly on the annular side of the mitral isthmus both endocardially and epicardial from within the CS. As previously described and expected for anatomical reasons, the main impact of VOM-EI is on the pulmonary venous side of the mitral isthmus, sparing the annular aspect. ${ }^{9}$ The pulmonary venous side of the mitral isthmus is generally thicker and protected from endocardial radiofrequency ablation by adipose tissue. ${ }^{17,18}$ Epicardial ablation via the CS is often required for mitral isthmus ablation. Because of the course of the CS, epicardial ablation usually targets the annular side of the mitral isthmus. VOM-EI can elegantly overcome the limitations of radiofrequency ablation by targeting primarily the pulmonary venous side. Correspondingly, several reports from the Bordeaux group demonstrated that endocardial radiofrequency ablation at the annular aspect of the mitral isthmus was mainly required to achieve mitral isthmus block after VOM-EI. ${ }^{13,19}$ The ablation time to achieve mitral isthmus block was significantly shorter with supplement VOM-EI compared to radiofrequency ablation alone. Termination of ongoing perimitral flutter during VOM-EI has been reported in 26-56\% of cases. ${ }^{9,10,15}$ Consistent with these results, perimitral flutter terminated or slowed during VOM-EI in $58 \%$ of our cases.

Radiofrequency ablation of atrial fibrillation increases cardiac biomarkers like hs-TnT. Haegeli et al. reported hs-TnT levels of $850 \mathrm{ng} / \mathrm{L}$ six hours after atrial fibrillation ablation whereas Reichlin et al. observed hs-TnT levels of $1996 \mathrm{ng} / \mathrm{L} 24$ hours after the procedure and found a significant correlation of hs-TnT levels with total radiofrequency time and energy delivery. ${ }^{20,21} \mathrm{In}$ our study, hs-TnT increased significantly from 330 to $598 \mathrm{ng} / \mathrm{L}$ within 24 hours after the procedure. Tissue necrosis induced by VOM-EI is therefore not excessive, as compared to a regular atrial fibrillation ablation procedure.

Low-voltage areas induced by VOM-EI can have various sizes, and probably depend on the vascular tributaries of the vein of Marshall as well as volume and infusion rate of ethanol injection. ${ }^{9,22}$ Some cases of chemical ablation have been reported that resulted in large low-voltage areas, including the left atrial appendage or the posterior wall. ${ }^{23,24}$ In previous studies, mean low-voltage areas induced by VOM-EI have been described in the range of $7.7 \pm 3.2 \mathrm{~cm}^{2}$ to $12.7 \pm 8.3 \mathrm{~cm}^{2}{ }^{8,9,10,25}$ Generally, 2 to $4 \mathrm{ml}$ of ethanol were injected in these studies, but some groups reported higher volumes of ethanol injection up to $12 \mathrm{ml} \cdot{ }^{10}$ After 
injection of a median of $4 \mathrm{ml}$ of ethanol, we observed a low-voltage area of $13.2 \mathrm{~cm}^{2}$. Low-voltage area in our study correlated significantly with the volume of ethanol injected $(\mathrm{P}=0.03)$.

\section{3 | Mitral isthmus block durability}

Previous reports described improved durability and lower arrhythmia recurrence after VOM-EI facilitated mitral isthmus block. Liu et al. stratified 254 patients into three groups: 1) VOM-EI with supplement pulmonary vein isolation with substrate modification; 2) pulmonary vein isolation with substrate modification; and 3) pulmonary vein isolation alone. ${ }^{15}$ They demonstrated a significant lower atrial arrhythmia recurrence rate in the VOM-EI group in comparison with pulmonary vein isolation with substrate modification or pulmonary vein isolation alone $(28.1 \%$ vs. $54.7 \%$ vs. $43.8 \%, \mathrm{P}=0.018)$. Another study by Takigawa et al. reported a better outcome of perimitral flutter treated by VOM-EI than by radiofrequency alone at one year and a reduction of atrial tachycardia recurrence rate of $65 \%$ in the VOM-EI group compared to the radiofrequency ablation group. ${ }^{19}$

The Bordeaux group described mitral isthmus line reconnection in $37.1 \%$ of the patients with redo procedures after VOM-EI. ${ }^{13}$ We found a reconnected mitral isthmus line in three out of five patients with recurrent arrhythmia (60\%). The gap on the mitral isthmus line was located on the annular side of the mitral isthmus in two out of three patients $(66 \%)$, where VOM-EI is ineffective and additional radiofrequency ablation is necessary to achieve mitral isthmus block.

Evolution of the low-voltage area induced by VOM-EI during follow-up showed shrinking of the low-voltage area in four out of five patients. Although a meaningful analysis was not possible because of the low number of patients, it is reassuring that the low-voltage area induced by VOM-EI remained stable and did not expand unpredictably during follow-up. Further studies are needed to analyse the correlation between induced lowvoltage area and the rate of arrhythmia recurrence after VOM-EI.

\section{5 | CONCLUSION}

Chemical ablation of VOM is feasible and safe. In $14 \%$ of cases, VOM-EI was not possible. With ancillary radiofrequency ablation, acute mitral isthmus block can be achieved in all patients.

\section{REFERENCES}

1. Jaïs P, Hocini M, Hsu LF, Sanders P, Scavee C, Weerasooriya R, Macle L, Raybaud F, Garrigue S, Shah DC, Metayer P Le, Clémenty J, Haïssaguerre M. Technique and results of linear ablation at the mitral isthmus. Circulation . 2004;110:2996-3002.

2. Fassini G, Riva S, Chiodelli R, Trevisi N, Berti M, Carbucicchio C, Maccabelli G, Giraldi F, Bella P Della. Left mitral isthmus ablation associated with PV isolation: Long-term results of a prospective randomized study. J Cardiovasc Electrophysiol . 2005;16:1150-1156.

3. Hocini M, Shah AJ, Nault I, Rivard L, Linton N, Narayan S, Myiazaki S, Jadidi AS, Knecht S, Scherr D, Wilton SB, Roten L, Pascale P, Pedersen M, Derval N, Sacher F, Jaïs P, Clémenty J, Haïssaguerre M. Mitral Isthmus Ablation with and Without Temporary Spot Occlusion of the Coronary Sinus: A Randomized Clinical Comparison of Acute Outcomes.J Cardiovasc Electrophysiol . 2012;23:489-496.

4. Haïssaguerre M, Hocini M, Takahashi Y, O’Neill MD, Pernat A, Sanders P, Jonsson A, Rotter M, Sacher F, Rostock T, Matsuo S, Arantés L, Teng Lim K, Knecht S, Bordachar P, Laborderie J, Jaïs P, Klein G, Clémenty J. Impact of catheter ablation of the coronary sinus on paroxysmal or persistent atrial fibrillation. J Cardiovasc Electrophysiol . 2007;18:378-386.

5. Yokokawa M, Sundaram B, Garg A, Stojanovska J, Oral H, Morady F, Chugh A. Impact of mitral isthmus anatomy on the likelihood of achieving linear block in patients undergoing catheter ablation of persistent atrial fibrillation. Heart Rhythm . 2011;8:1404-1410.

6. Han S, Joung B, Scanavacca M, Sosa E, Chen P-S, Hwang C. Electrophysiological characteristics of the Marshall bundle in humans. Heart Rhythm . 2010;7:786-793. 
7. Valderrabano M, Chen HR, Sidhu J, Rao L, Ling Y, Khoury DS. Retrograde Ethanol Infusion in the Vein of Marshall. Circ Arrhythmia Electrophysiol . 2009;2:50-56.

8. Valderrábano M, Liu X, Sasaridis C, Sidhu J, Little S, Khoury DS. Ethanol infusion in the vein of Marshall: Adjunctive effects during ablation of atrial fibrillation. Heart Rhythm . 2009;6:1552-1558.

9. Báez-Escudero JL, Morales PF, Dave AS, Sasaridis CM, Kim Y-H, Okishige K, Valderrábano M. Ethanol infusion in the vein of Marshall facilitates mitral isthmus ablation. Heart Rhythm . 2012;9:1207-1215.

10. Kitamura T, Vlachos K, Denis A, Andre C, Martin R, Pambrun T, Duchateau J, Frontera A, Takigawa M, Thompson N, Cheniti G, Martin CA, Lam A, Bourier F, Sacher F, Hocini M, Haissaguerre M, Jais P, Derval N. Ethanol infusion for Marshall bundle epicardial connections in Marshall bundle-related atrial tachycardias following atrial fibrillation ablation: The accessibility and success rate of ethanol infusion by using a femoral approach. J Cardiovasc Electrophysiol . 2019;30:1443-1451.

11. Valderrabano M, Peterson LE, Swarup V, Schurmann PA, Makkar A, Doshi RN, DeLurgio D, Athill CA, Ellenbogen KA, Natale A, Koneru J, Dave AS, Giorgberidze I, Afshar H, Guthrie ML, Bunge R, Morillo CA, Kleiman NS. Effect of Catheter Ablation With Vein of Marshall Ethanol Infusion vs Catheter Ablation Alone on Persistent Atrial Fibrillation. JAMA . 2020;324:1620.

12. Hwang C, Wu T-J, Doshi RN, Peter CT, Chen P-S. Vein of Marshall Cannulation for the Analysis of Electrical Activity in Patients With Focal Atrial Fibrillation. Circulation . 2000;101:1503-1505.

13. Nakashima T, Pambrun T, Vlachos K, Goujeau C, Andre C, Krisai P, Ramirez FD, Kamakura T, Takagi T, Nakatani Y, Kitamura T, Takigawa M, Roux J-R, Cheniti G, Tixier R, Chauvel R, Welte N, Duchateau J, Sacher F, Cochet H, Hocini M, Haissaguerre M, Jais P, Derval N. Impact of Vein of Marshall Ethanol Infusion on Mitral Isthmus Block: Efficacy and Durability. Circ Arrhythmia Electrophysiol . 2020;CIRCEP.120.008884.

14. Kawaguchi N, Okishige K, Yamauchi Y, Kurabayashi M, Nakamura T, Keida T, Sasano T, Hirao K, Valderrabano M. Clinical impact of ethanol infusion into the vein of Marshall on the mitral isthmus area evaluated by atrial electrograms recorded inside the coronary sinus. Heart Rhythm . 2019;16:1030-1038.

15. Liu CM, Lo LW, Lin YJ, Lin CY, Chang SL, Chung FP, Chao TF, Hu YF, Tuan TC, Liao JN, Chen YY, Kuo L, Chang TY, Hoang QM, Salim S, Vicera JJB, Wu CI, Chuang CM, Huang TC, Chen SA. Longterm efficacy and safety of adjunctive ethanol infusion into the vein of Marshall during catheter ablation for nonparoxysmal atrial fibrillation. J Cardiovasc Electrophysiol . 2019;30:1215-1228.

16. Kawaguchi N, Okishige K, Yamauchi Y, Kurabayashi M, Nakamura T, Keida T, Sasano T, Hirao K, Valderrabano M. Clinical Impact of an Ethanol Infusion into the Vein of Marshall on the Mitral Isthmus Area evaluated by Atrial Electrograms recorded inside the Coronary Sinus.Heart Rhythm . 2019;16:1030-1038.

17. Becker AE. Left atrial isthmus: Anatomic aspects relevant for linear catheter ablation procedures in humans. J Cardiovasc Electrophysiol . 2004;15:809-812.

18. West JJ, Norton PT, Kramer CM, Moorman JR, Mahapatra S, DiMarco JP, Mangrum JM, Mounsey JP, Ferguson JD. Characterization of the mitral isthmus for atrial fibrillation ablation using intracardiac ultrasound from within the coronary sinus. Heart Rhythm . 2008;5:19-27.

19. Takigawa M, Vlachos K, Martin CA, Bourier F, Denis A, Kitamura T, Cheniti G, Lam A, Martin R, Frontera A, Thompson N, Massoullie G, Wolf M, Escande W, Andre C, Zeng LJ, Nakatani Y, Nakashima T, Pillois X, Ramirez D, Duchateau J, Pambrun T, Sacher F, Cochet H, Hocini M, Haissaguerre M, Jais P, Derval N. Acute and mid-term outcome of ethanol infusion of vein of Marshall for the treatment of perimitral flutter.Europace . 2020;22:1252-1260.

20. Haegeli LM, Kotschet E, Byrne J, Adam DC, Lockwood EE, Leather RA, Sterns LD, Novak PG. Cardiac injury after percutaneous catheter ablation for atrial fibrillation. Europace . 2008;10:273-275. 
21. Reichlin T, Lockwood SJ, Conrad MJ, Nof E, Michaud GF, John RM, Epstein LM, Stevenson WG, Jarolim P. Early release of high-sensitive cardiac troponin during complex catheter ablation for ventricular tachycardia and atrial fibrillation. J Interv Card Electrophysiol . 2016;47:69-74.

22. Pambrun T, Denis A, Duchateau J, Sacher F, Hocini M, Jais P, Haissaguerre M, Derval N. MARSHALL bundles elimination, Pulmonary veins isolation and Lines completion for ANatomical ablation of persistent atrial fibrillation: MARSHALL-PLAN case series. J Cardiovasc Electrophysiol . 2019;30:7-15.

23. Chugh A, Gurm HS, Krishnasamy K, Saeed M, Lohawijarn W, Hornsby K, Cunnane R, Ghanbari H, Latchamsetty R, Crawford T, Jongnarangsin K, Bogun F, Oral H, Morady F. Spectrum of atrial arrhythmias using the ligament of Marshall in patients with atrial fibrillation. Heart Rhythm . 2017;15:17-24.

24. Derval N, Duchateau J, Denis A, Ramirez FD, Mahida S, Andre C, Krisai P, Nakatani Y, Kitamura T, Takigawa M, Chauvel R, Tixier R, Pillois X, Sacher F, Hocini M, Haissaguerre M, Jais P, Pambrun T. Marshall bundle elimination, Pulmonary vein isolation, and Line completion for ANatomical ablation of persistent atrial fibrillation (Marshall-PLAN): Prospective, single-center study. Heart Rhythm . 2020;137421.

25. Dave AS, Baez-Escudero JL, Sasaridis C, Hong TE, Rami T, Valderrabano M. Role of the Vein of Marshall in Atrial Fibrillation Recurrences After Catheter Ablation: Therapeutic Effect of Ethanol Infusion. J Cardiovasc Electrophysiol . 2012;23:583-591.

\section{FIGURE LEGENDS}

\section{FIGURE 1}

Left posterior-caudal view showing a voltage map of the left atrium (CARTO 3, Biosense Webster). A) before and B) after ethanol infusion into the VOM

\section{FIGURE 2}

A) Angiography (right anterior oblique 30deg view) of the great cardiac vein and vein of Marshall (arrow).

B) Selective angiography of the VOM after proximal balloon occlusion (arrow)

\section{FIGURE 3}

Selective angiography of the VOM (arrow), overlaid by a voltage map of the left atrium in left posteriorcaudal view, showing the corresponding low-voltage area after VOM-EI (CARTO 3, Biosense Webster)

\section{FIGURE 4}

Termination of peri-mitral flutter 42 seconds after ethanol infusion into the VOM. The Pentaray catheter is placed in the left atrial appendage; 20PA 1 to 20: Pentaray electrodes; II, V1 and V5: body surface ECG leads. Sensis/ Syngo Dynamics (Siemens Healthcare GmbH, Germany)

\section{FIGURE 5}

Correlation of the volume of ethanol injected and the low-voltage area in the VOM region

\section{FIGURE 6}

CK, CK-MB and hs-TnT in the evening of the procedure and on the morning following VOM-EI. CK, Creatine Kinase; CK-MB, Creatine Kinase Myocardial Band; hs-TnT, high-sensitive cardiac troponin-T

\section{FIGURE 7}

Selective angiography (right anterior oblique 30deg view) in a patient with two veins in the VOM region (arrows)

\section{FIGURE 8}

Low-voltage area at the end of the index procedure (A) in comparison to the low-voltage area at the beginning of the five redo cases (B). 


\section{TABLES}

TABLE 1. Patient characteristics

\begin{tabular}{ll}
\hline Total & $\mathbf{n = 2 2}$ \\
\hline Male & $14(64 \%)$ \\
Age, years & $71(55-74)$ \\
Left ventricular ejection fraction, $\%$ & $57.5(43.8-60.0)$ \\
Indication for VOM-EI & $17(77 \%)$ \\
Perimitral flutter & $4(18 \%)$ \\
Mitral isthmus line ablation for persistent atrial & \\
fibrillation & $1(5 \%)$ \\
VOM tachycardia & $18(82 \%)$ \\
Previous ablation in left atrium & $2.0(1.0-2.0)$ \\
Number of previous ablation per patient & $18(100 \%)$ \\
Pulmonary vein isolation & $7(39 \%)$ \\
Roof line & $4(22 \%)$ \\
Mitral isthmus line & $2(50 \%)$ \\
Mitral isthmus block achieved & $2(11 \%)$ \\
Defragmentation in left atrium & $2(9 \%)$ \\
Superior vena cava isolation & $12(55 \%)$ \\
Cavotricuspid isthmus line ablation &
\end{tabular}

Shown are numbers with percentages in parentheses or medians with interquartile range.

$\mathrm{VOM}=$ vein of Marshall, VOM-EI = ethanol infusion into the VOM

TABLE 2. Procedural characteristics of patients with VOM ethanol infusion

\begin{tabular}{ll}
\hline Total & $\mathbf{n = 1 9}$ \\
\hline CS angiography with LIMA catheter & $19(100 \%)$ \\
Balloon size, mm & $2.0(1.5-2.0)$ \\
Volume of ethanol injected, ml & $4.0(3.0-6.0)$ \\
Rhythm during VOM-EI & $3(16 \%)$ \\
Sinus rhythm & $3(16 \%)$ \\
Atrial fibrillation & $12(63 \%)$ \\
Atrial flutter & $4(33 \%)$ \\
Termination to sinus rhythm during VOM-EI & $3(25 \%)$ \\
Cycle length prolongation during VOM-EI & $1(5 \%)$ \\
Paced rhythm & $8(42 \%)$ \\
Radiofrequency ablation of mitral isthmus line & \\
before VOM-EI & $7(37 \%)$ \\
Mitral isthmus block with VOM-EI & $12(63 \%)$ \\
Radiofrequency ablation of mitral isthmus line & \\
after VOM-EI & \\
Other Radiofrequency ablation targets during & \\
VOM-EI procedure & $11(58 \%)$ \\
Pulmonary vein isolation & $8(42 \%)$ \\
Roof line & $1(5 \%)$ \\
Defragmentation in left atrium & $2(11 \%)$ \\
Superior vena cava isolation &
\end{tabular}




\begin{tabular}{ll}
\hline Total & $\mathbf{n = 1 9}$ \\
\hline Cavotricuspid isthmus line & $4(21 \%)$ \\
Adverse events & $1(5 \%)$ \\
Pericarditis & $3(16 \%)$ \\
Minor pericardial effusion & $2(11 \%)$ \\
VOM dissection & $1(5 \%)$ \\
Symptomatic sinus arrest &
\end{tabular}

Shown are numbers with percentages in parentheses or medians with interquartile range.

$\mathrm{CS}=$ coronary sinus, LIMA = left internal mammary artery, VOM = vein of Marshall, VOM-EI $=$ ethanol infusion into the VOM

TABLE 3. Follow-up and redo procedures

\begin{tabular}{ll}
\hline Total & $\mathbf{n = 1 8}$ \\
\hline Time to follow-up after VOM-EI, months & $3.5(3.0-11.0)$ \\
Follow-up device & $11(61 \%)$ \\
Holter-ECG & $7(39 \%)$ \\
ICD, pacemaker or ILR & $10(56 \%)$ \\
Recurrences & $6(60 \%)$ \\
Atrial fibrillation & $4(40 \%)$ \\
Atrial flutter & $5(50 \%)$ \\
Redo procedures after VOM-EI & $3(60 \%)$ \\
Mitral isthmus line reablation & \\
\hline
\end{tabular}

Shown are numbers with percentages in parentheses or medians with interquartile range. $\mathrm{P}$ values are two-sided, with a $\mathrm{P}<0.05$ considered to indicate statistical significance.

VOM-EI = ethanol infusion into the vein of Marshall, ECG $=$ electrocardiogram, ICD = implantable cardiac defibrillator, ILR = implantable loop recorder

\section{FIGURES}

FIGURE 1

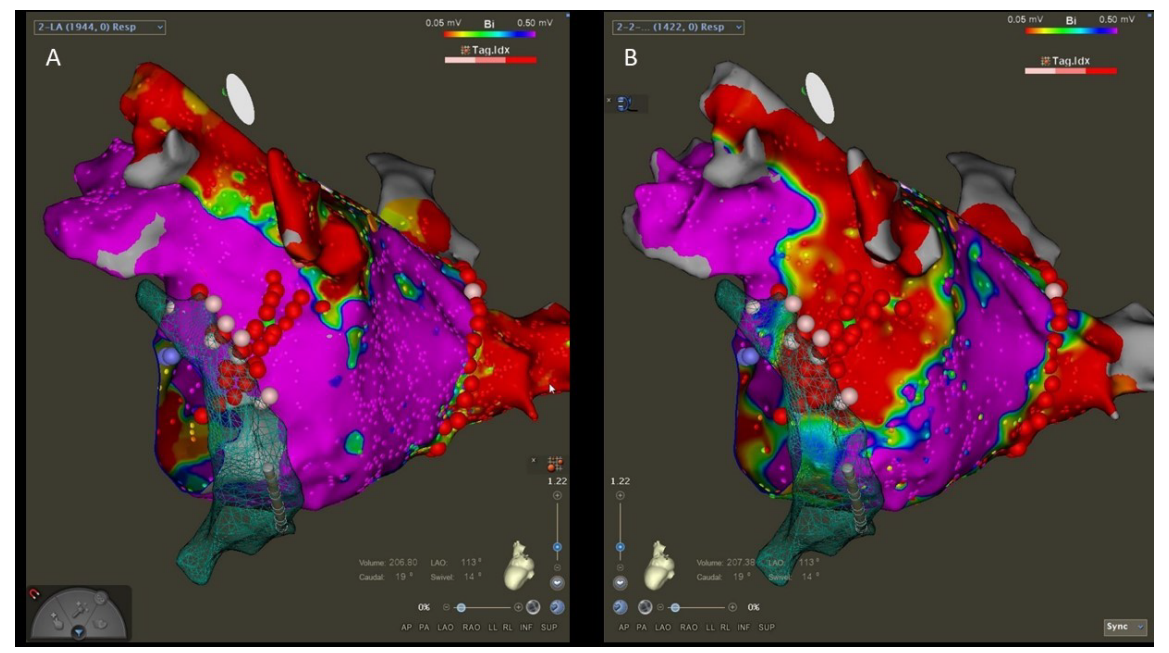


FIGURE 2

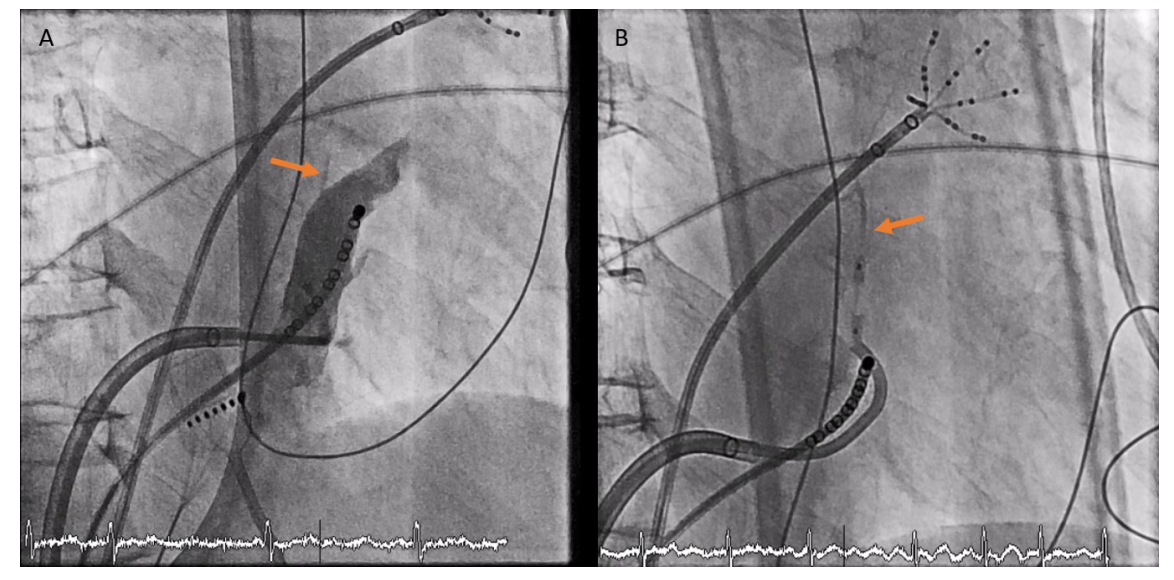

FIGURE 3

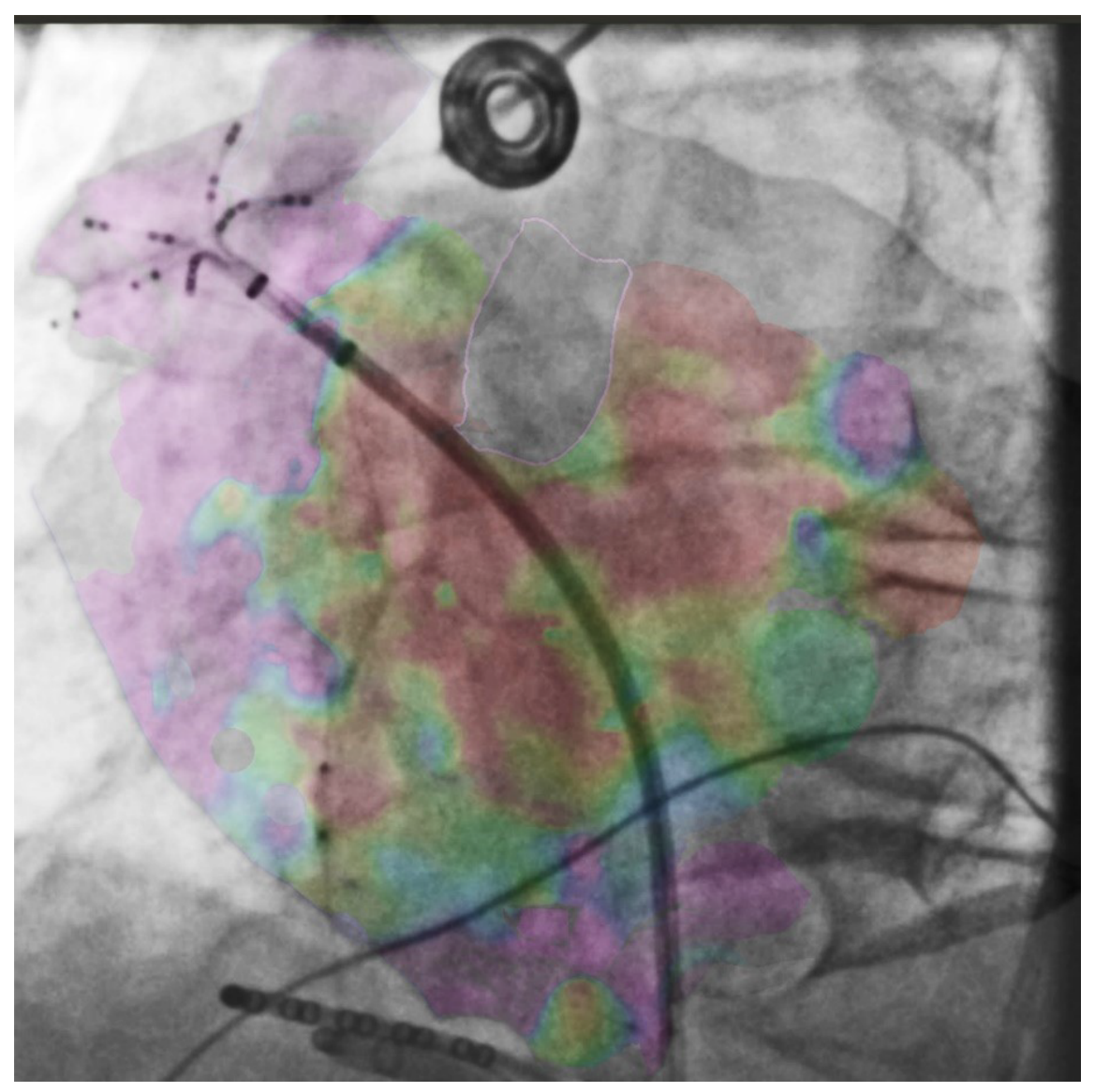

FIGURE 4 


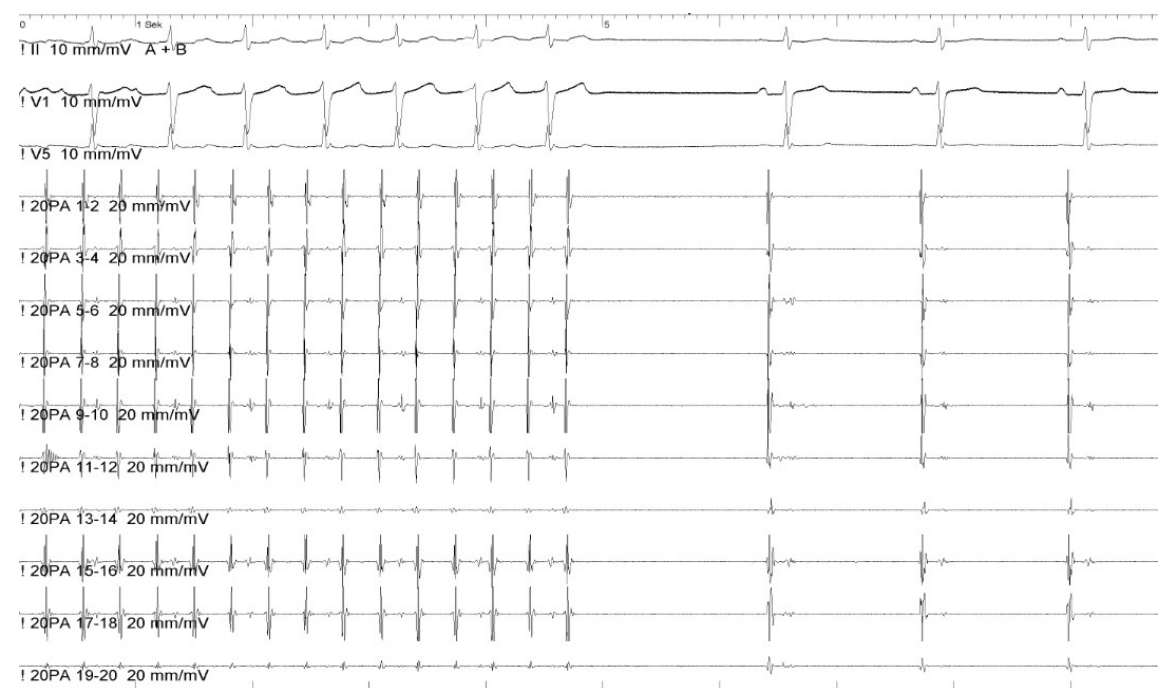

FIGURE 5
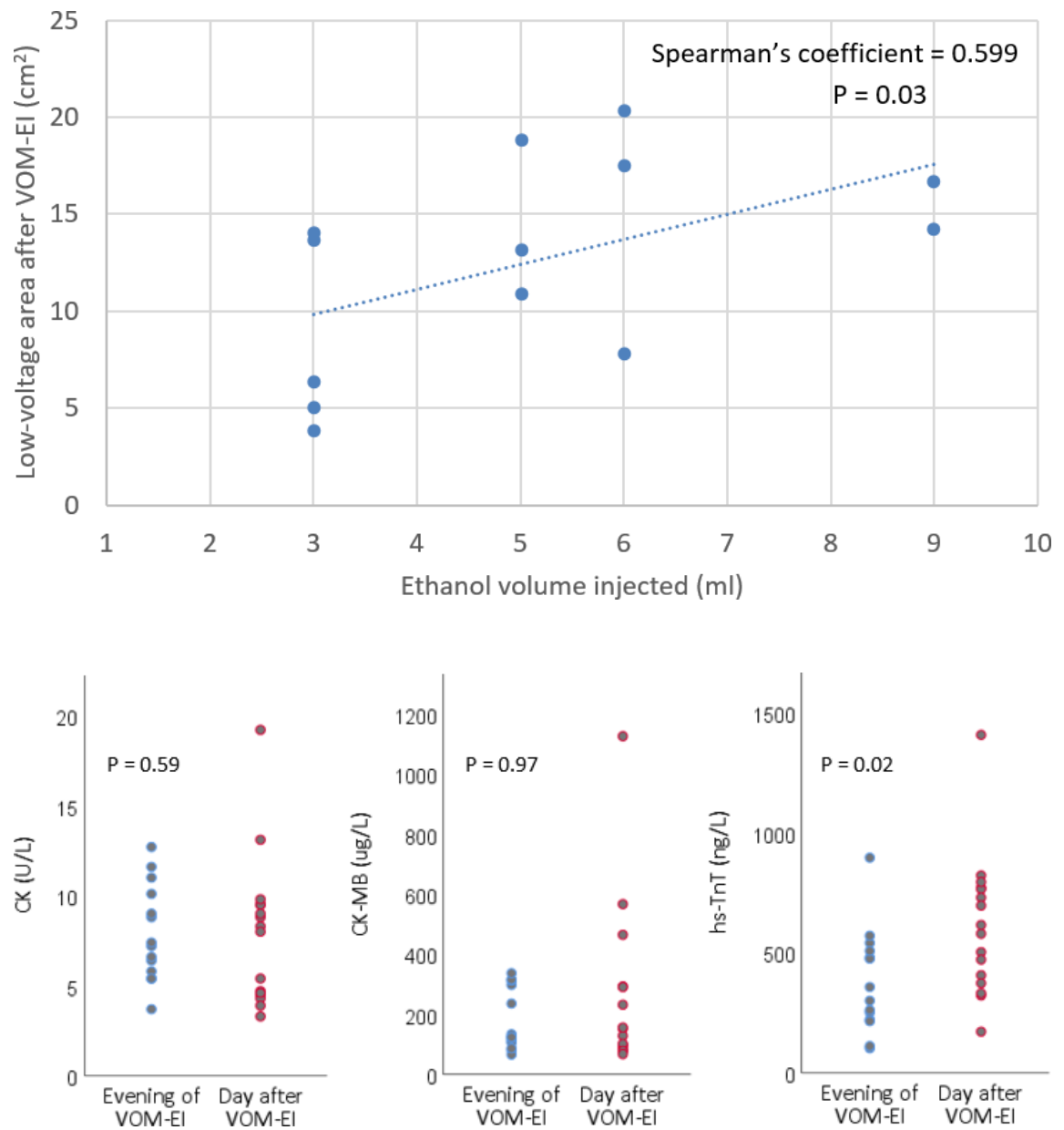

FIGURE 6 
FIGURE 7

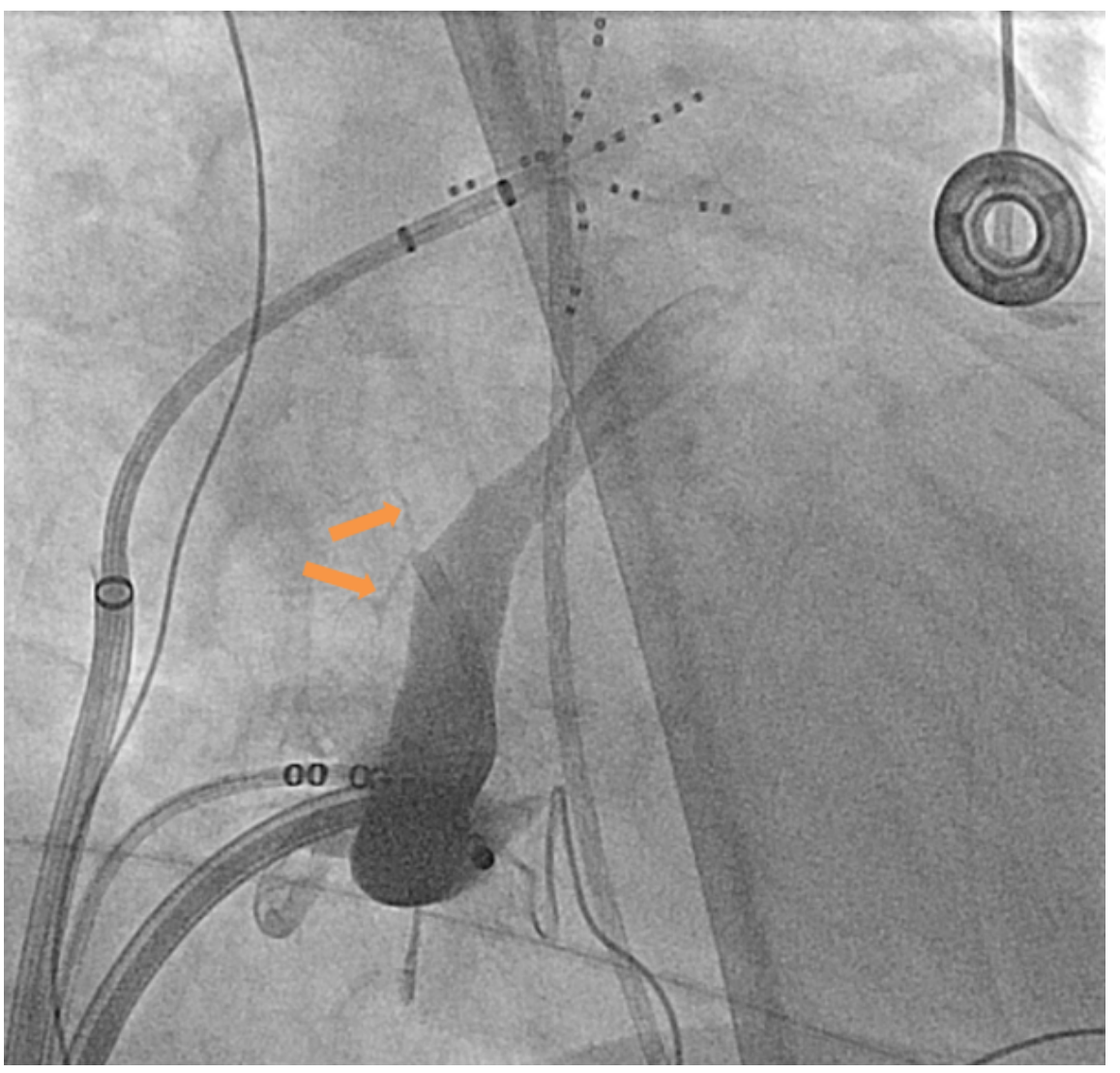




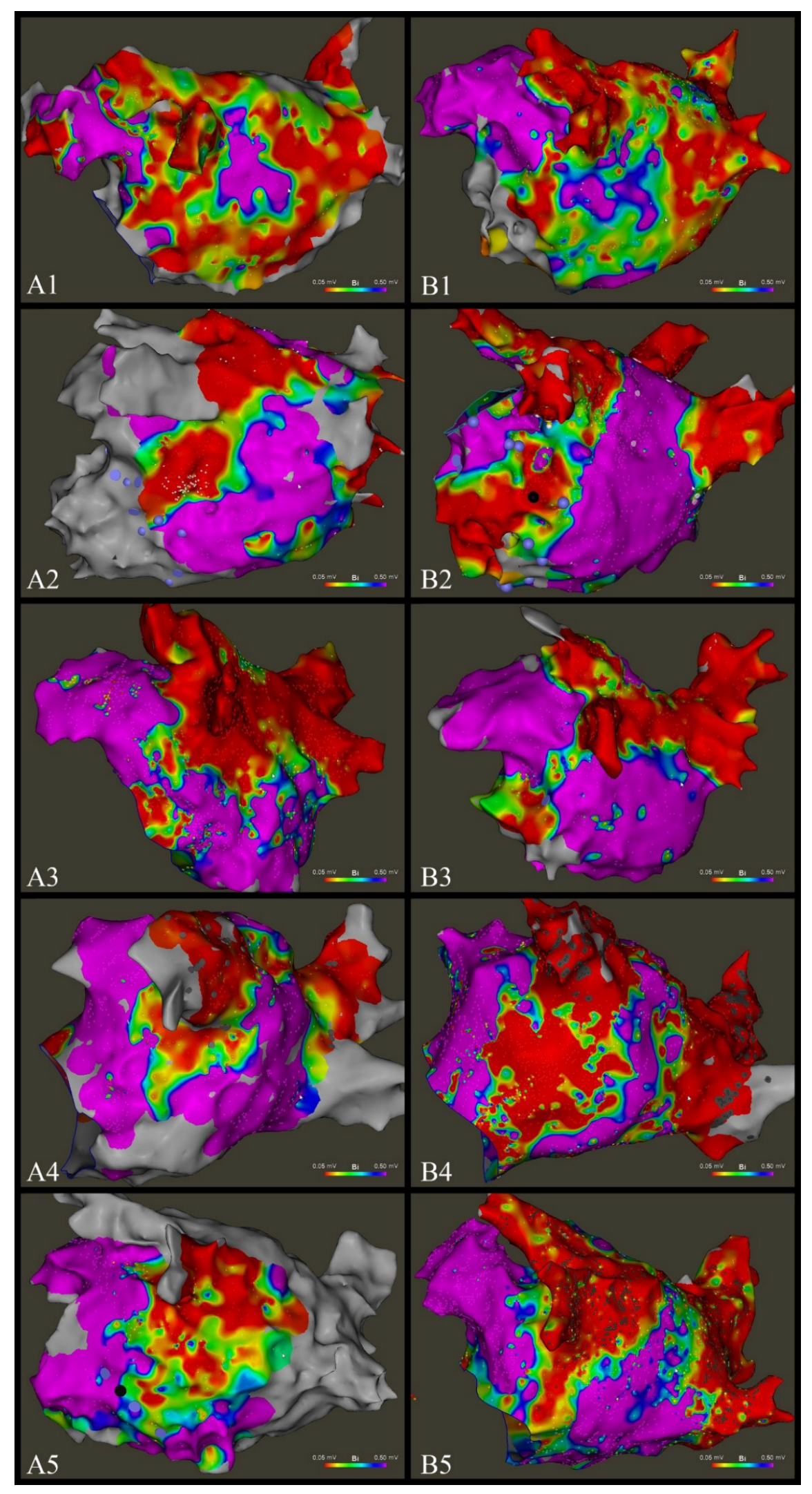


FIGURE 8 